\title{
Light-Like Shockwaves in Scalar-Tensor Theories
}

\author{
Bence Racskó ${ }^{1, *(1)}$ and László Árpád Gergely 2 (10) \\ 1 Institute of Physics, University of Szeged, H-6720 Szeged, Hungary \\ 2 Institute of Physics, University of Szeged, H-6720 Szeged, Hungary; laszlo.a.gergely@gmail.com \\ * Correspondence: daeron806@gmail.com; Tel.: +36-305535764
}

Received: 10 December 2017; Accepted: 22 February 2018; Published: 27 February 2018

\begin{abstract}
Both electromagnetic shock-waves and gravitational waves propagate with the speed of light. If they carry significant energy-momentum, this will change the properties of the space-time they propagate through. This can be described in terms of the junction conditions between space-time regions separated by a singular, null hypersurface. We derived generic junction conditions for Brans-Dicke theory in the Jordan frame, exploring a formalism based on a transverse vector, rather than normal, which can be applied to any type of hypersurfaces. In the particular case of a non-null hypersurface we obtain a generalised Lanczos equation, in which the jump of the extrinsic curvature is sourced by both the distributional energy-momentum tensor and by the jump in the transverse derivative of the scalar. In the case of null hypersurfaces, the distributional source is decomposed into surface density, current and pressure. The latter, however, ought to vanish by virtue of the scalar junction condition.
\end{abstract}

Keywords: space-time junction conditions; Brans-Dicke theory; null hypersurfaces

\section{Introduction}

General relativity (GR) has withstood the confrontation with observations both in Solar System tests and in strong field regimes, the latter of which had been the experimental detection of gravitational waves by the LIGO Scientific Collaboration and Virgo Collaboration [1-6]. The direct detection of gravitational waves from black hole binaries and a neutron star coalescence has confirmed (through the test of the dispersion relations and of the model dependent delay in the arrival time of the gamma radiation following the neutron star merger) that they propagate with the speed of light $[3,6-8]$. In consequence, many modified/extended theories of gravitation were disruled. Nevertheless, there are still many interesting such theories, which allow for light-like gravitational wave propagation, that are still worth investigating.

General relativity (GR) has given predictions on both galactic scales and beyond which can be reconciled with observations only at the price of introducing still undetected (otherwise than gravitationally) dark matter and dark energy. There is hope that modified gravity theories might replace them by corresponding geometrical effects. Such modified gravity theories encompass either a more complicated (higher-order) dynamics for the metric tensor (but this may lead to instabilities and ghosts), or increase the number of the fields describing pure gravity, with adding scalars, vectors, 2 -form fields or even a second metric. The main difference as compared to models with additional fields representing the dark sector is in the way the metric couples to them: for the dark sector the coupling is minimal, for a geometric field it may be more complicated. The simplest of them would be a scalar-tensor theory. Horndeski has established $[9,10]$ the most generic class of such theories with both the metric tensor and the scalar field obeying second-order dynamics.

Historical interest in scalar-tensor theories of gravity began with the Kaluza-Klein theory. In 1919 Kaluza sought to unify gravity with electrodynamics by considering a five-dimensional spacetime, whose metric is subject to the Einstein field equations. To account for the observed four-dimensional 
nature of space-time, he assumed that the extra dimension is compact and small, hence the dependence on this fifth coordinate could be averaged out. Then, the five dimensional metric is decomposed into a four dimensional metric (describing gravity), a four-vector (describing electromagnetism), and a scalar field. At the time, the scalar field was thought to be undesirable, however later on (especially when the connection between fields and particles had been established), Kaluza-Klein theory proved to be an inspiration for more general theories of gravity with added scalar fields, such as the much-investigated Brans-Dicke theory.

In scalar-tensor theories it is customary to explore one of two conformally related metrics $g_{\mu \nu}^{\prime}(x)=\Omega^{2}(x) g_{\mu v}(x)$ (with $\Omega(x)$ a nowhere-vanishing smooth function): (1) the Jordan (JordanFierz or string) frame $g_{\mu v}$, in which the scalar field $\phi$ is coupled non-minimally to gravity, but is not coupled to matter fields, and (2) the Einstein frame $g_{\mu v}^{\prime}$, in which the coupling between gravity and the scalar field is minimal, but there is an anomalous coupling of the scalar field to the matter fields [11].

Despite scalar-tensor theories being around for a long time, there is still a heated debate on whether both frames are physical or not, and if so, whether they are physically equivalent. Dicke argued [12] that since physics must be invariant under the rescaling of units, and the conformal transformation is merely a local rescaling of distances, physics should not depend on the conformal frame, provided that the units of length, time and mass scale appropriately [13], although this argument has been criticized [11]. Some authors argue in favour of the Einstein frame, as in it comparison with pure GR results is easier [14] and the energy conditions for the scalar field are obeyed, while in the Jordan frame the scalar field violates all known energy conditions $[15,16]$. Other authors prefer the Jordan frame, satisfying the equivalence principle, which is violated in the Einstein frame due to the anomalous scalar field-matter couping. Therefore, in the Einstein frame, the matter stress-energy tensor rather than obeying a continuity equation is subject to $\nabla_{\mu} T^{\mu \nu}=-T \nabla^{v} \ln \Omega[11,14]$.

Both in GR and in modified gravity theories it is of special interest to match spacetime regions with different matter sources or even different set of symmetries. This can be done along a common hypersurface, which may be temporal, spatial or even null. Moreover, the hypersurface may contain a distributional energy-momentum layer, complicating the junction conditions. For GR they were worked out covariantly by Israel [17], but this formalism does not apply for null hypersurfaces. In order to deal with them, Barrabès and Israel proposed a modified junction formalism [18], relying on the use of a transverse vector to the null hypersurface.

Junction conditions can also be derived by employing a variational principle, both for GR and scalar-tensor theories in the Einstein frame (incorporating the scalars in the matter sector) [19]. The dynamics of bubbles (infinitesimally thin shells) or plane domain walls were considered in Brans-Dicke theory in the Jordan frame [20-23]. For the Horndeski class of theories they were discussed in the Jordan frame in [24], nevertheless only for space-like or time-like hypersurfaces and further applied in a cosmological setup [25]. These results, however, cannot be applied for null hypersurfaces. Such hypersurfaces may be of physical interest as they represent light-like shock-waves, both electromagnetic or gravitational, which modify the gravitational properties of spacetime they propagate through.

In this paper, we investigate a general approach for the junction conditions for scalar-tensor theories, which can be applied for any type of hypersurfaces. We opt for the Jordan frame, motivated by the desire to keep the generic form of the function $G_{4}$ in the Hordeski Lagrangian. Another motivation for assuming that the sources couple to gravity only via the metric tensor, and not via the scalar field would be to avoid any non-gravitational interaction of the scalar field with the baryonic matter fields $\psi^{i}$, hence to be able to describe the dark sector with $\phi$. We derive the Euler-Lagrange equations for both the metric and the scalar and investigate the singular contributions, which would appear only in the second derivatives of both fields (terms proportional to Dirac-delta functions). This is related to the approach of [26], which considers metrics whose curvature tensors are well defined as distributions (e.g., avoid products of distributions). 
We derive the junction equations by separating the singular contributions to the field equations and connecting them to distributional sources in Section 2. As a first exercise we apply the formalism for the Brans-Dicke scalar-tensor theory in Section 3. We note that a related treatment was developed in [14], but in Einstein frame and for multi-scalar fields. Finally in Section 4 we specify our results for null hypersurfaces, which is followed by a Summary.

\section{Junction Conditions across Arbitrary Hypersurfaces}

We consider the spacetime $M$ cut into two disjoint parts $M^{+}$and $M^{-}$by a common boundary hypersurface $\Sigma$, with unspecified causal character. We allow certain otherwise smooth geometric quantities to undergo sudden changes at the boundary, leading to discontinuities across $\Sigma$. Physically, $\Sigma$ may separate a star from its exterior (collapsing stars included); however, it also can be the world-volume of a shockwave (for example, one emanating from a supernova explosion), or a space-like hypersurface encompassing a cosmological phase transition, among others.

Despite the junction, $M$ does possess a smooth structure [27]; hence, in principle it is possible to use a coordinate system that transitions smoothly across $\Sigma$. However, in practical situations, such coordinate systems might not be straightforward to identify, hence, we will express all equations on the junction surface in coordinate charts internal to $\Sigma$. In other words we use a doubly covariant formalism.

Null hypersurfaces representing shockwaves travelling at the speed of light are physically relevant, nevertheless their study is obstructed by the fact that they have degenerate metrics and their normal vectors are also tangential, preventing a proper orthogonal decomposition of quantities along $\Sigma$. Therefore, following [18] we explore an oblique decomposition, valid for all types of hypersurfaces. The holonomic basis vectors of the hypersurface are denoted $e_{a}^{\mu}$, with a transverse vector field $l^{\mu}$ completing the basis. The normal covector field $n_{\mu}$ satisfies $n_{\mu} l^{\mu}=1 / \eta(\eta$ arbitrary and nonvanishing) with the norm $\epsilon=n^{\mu} n_{\mu}$ (depending on the type of hypersurface, $\epsilon= \pm 1,0$ ). If $\Sigma$ is given as the zero set of a scalar field $f$, then $n_{\mu}=\frac{1}{\alpha} \partial_{\mu} f$, with $\alpha$ a normalising factor.

For an arbitrary field quantity $F$ on $M$, its jump, arithmetic mean and soldering accross $\Sigma$ are [18]:

$$
\begin{aligned}
{[F] } & =F^{+}-\left.F^{-}\right|_{\Sigma}, \\
\bar{F} & =\left.\frac{1}{2}\left(F^{+}+F^{-}\right)\right|_{\Sigma}, \\
\widetilde{F} & =F^{+} \Theta(f)+F^{-} \Theta(-f),
\end{aligned}
$$

with

$$
\Theta(x)=\left\{\begin{array}{l}
1, x>0 \\
0, x<0 \\
\frac{1}{2}, x=0
\end{array}\right.
$$

the Heaviside function. It is straightforward to derive the relation

$$
\partial_{\mu} \widetilde{F}=\widetilde{\partial_{\mu} F}+[F] n_{\mu} \alpha \delta(f) .
$$

In a scalar-tensor theory with at most second-order dynamics (Horndeski class [9], avoiding Ostrogradsky-instabilities [28], see also [29]) we require that none of the contributions to the field equations exhibit derivatives of Dirac-delta functions (difficult to interpret from a physical point of view), while the Dirac-delta functions themselves will be allowed (related to the density of some finite quantity characterising an idealised, infinitely thin layer along $\Sigma$ ). This condition can be assured by assuming both $g_{\mu \nu}$ (in smooth coordinates) and $\phi$ continuous across $\Sigma$. The continuity of the induced metric is the first junction condition of Israel [17]. The continuity of the rest of the metric components can be assured by picking up $C^{1}$ Gaussian normal coordinates, the existence of which has been proven in [27]. 
The action of the system is

$$
\begin{aligned}
S\left[g_{\mu v}, \phi, \psi^{i}\right] & =S_{G}\left[g_{\mu v}, \phi\right]+S_{M}\left[g_{\mu v}, \psi^{i}\right], \\
S_{G}\left[g_{\mu v}, \phi\right] & =\int d^{4} x\left(\sum_{k=2}^{5} L_{k}\right),
\end{aligned}
$$

with the gravitational part given by the Horndeski Lagrangians and the matter part independent of $\phi$ in order to assure that the equivalence principle remains valid (this argument applies to the Jordan frame, which is the physical frame, where energy-momentum conservation holds).

We note that the recent confirmation of the gravitational wave propagation speed to agree with the speed of light at the order of one part in quadrillionth $[7,8]$ at low redshifts has disruled theories with dependence of the kinetic term $X=-\frac{1}{2} \nabla^{\mu} \phi \nabla_{\mu} \phi$ in the coupling of the Ricci curvature $R$ and Einstein tensor $G_{\mu v}$ in $L_{4}$ and $L_{5}$, respectively [30,31]. Further, the latter does not depend on $\phi$ either (except through its derivatives), hence, due to the Bianchi identities, the whole $L_{5}$ ought to vanish [32] (see also [33,34]).

The energy-momentum tensor associated with the matter fields is defined as

$$
T_{\mu \nu}=-\frac{2}{\sqrt{-g}} \frac{\delta S_{M}}{\delta g^{\mu \nu}} .
$$

Without specifying the details of the dynamics, we can denote the left hand sides (lhs) of the Euler-Lagrange equations as

$$
E_{\mu \nu}=\frac{1}{\sqrt{-g}} \frac{\delta S_{G}}{\delta g^{\mu \nu}}
$$

and

$$
E^{\phi}=\frac{1}{\sqrt{-g}} \frac{\delta S_{G}}{\delta \phi},
$$

the equations of motion being

$$
E_{\mu v}=\frac{1}{2} T_{\mu v}, \quad E^{\phi}=0 .
$$

The lhs' exhibit the following dependencies:

$$
\begin{aligned}
E_{\mu \nu} & =E_{\mu \nu}\left(\phi, \partial \phi, \partial^{2} \phi, g, \partial g, \partial^{2} g\right), \\
E^{\phi} & =E^{\phi}\left(\phi, \partial \phi, \partial^{2} \phi, g, \partial g, \partial^{2} g\right) .
\end{aligned}
$$

Plugging in the continuous fields $g_{\mu \nu}=g_{\mu \nu}^{+} \Theta(f)+g_{\mu \nu}^{-} \Theta(-f)$ and $\phi=\phi^{+} \Theta(f)+\phi^{-} \Theta(-f)$, their first derivatives generate jumps, while the second derivatives terms proportional to $\delta(f)$ :

$$
\begin{aligned}
E^{\mu v} & =\widetilde{E}^{\mu v}+\mathscr{E}^{\mu v} \alpha \delta(f), \\
E^{\phi} & =\widetilde{E^{\phi}}+\mathscr{E}^{\phi} \alpha \delta(f) .
\end{aligned}
$$

Similarly, the energy-momentum tensor allows for a distributional contribution on $\Sigma$ :

$$
T^{\mu v}=\widetilde{T}^{\mu v}+\mathscr{T}^{\mu v} \alpha \delta(f) .
$$

The junction conditions are therefore the distributional equations of motion:

$$
\mathscr{E}^{\mu \nu}=\frac{1}{2} \mathscr{T}^{\mu \nu}, \quad \mathscr{E}^{\phi}=0,
$$

along with the continuity condition $[\phi]=\left[g_{\mu \nu}\right]=0$. 
The scalar Equation (11) is simple to be evaluated on $\Sigma$. The tensor Equation (11) can be decomposed with respect to the oblique basis, employing a $\Sigma$-scalar $\mathscr{E}_{l}$, a $\Sigma$-vector $\mathscr{E}_{l} a$ and a $\Sigma$-tensor $\mathscr{E}^{a b}$ defined as

$$
\mathscr{E}^{\mu \nu v}=\mathscr{E}_{l} l^{\mu} l^{v}+2 \mathscr{E}_{l}^{a} e_{a}^{(\mu} l^{v)}+\mathscr{E}^{a b} e_{a}^{\mu} e_{b}^{v} .
$$

This decomposition is left unchanged by coordinate transformations on $M^{ \pm}$. However, only the $\mathscr{E}^{a b}$ part would be nonvanishing, as the distributional stress-energy tensor $\mathscr{T}^{\mu v}$ represents the intrinsic stress energy of the singular source on the surface. In GR the vectorial and scalar contributions can be expressed in terms of the Hamiltonian and diffeomorphism constraints, hence they do not carry new information. The same has been verified for the simplest scalar-tensor theories. Therefore, the junction conditions can be rewritten as equations on $\Sigma$ as

$$
\mathscr{E}^{a b}=\frac{1}{2} \mathscr{T}^{a b}, \quad \mathscr{E} \phi=0 .
$$

\section{Brans-Dicke Theory}

Perhaps the most well-known scalar-tensor theory is the Brans-Dicke theory, born from the simple assumption of replacing the gravitational constant with a scalar field $\phi$. Its Lagrangian in the Jordan frame

$$
L_{B D}=-\frac{\omega}{16 \pi \phi} \partial^{\mu} \phi \partial_{\mu} \phi+\frac{R \phi}{16 \pi},
$$

contains a coupling constant $\omega$. The field equations obtained from the metric and scalar field variations are

$$
\begin{aligned}
8 \pi T_{\mu \nu} & =\frac{\omega}{\phi}\left[-\nabla_{\mu} \phi \nabla_{\nu} \phi+\frac{1}{2}(\nabla \phi)^{2} g_{\mu \nu}\right]+\phi G_{\mu \nu} \\
& -\nabla_{\mu} \nabla_{\nu} \phi+\square \phi g_{\mu \nu} \\
0 & =-\frac{\omega}{\phi}(\nabla \phi)^{2}+\phi R+2 \omega \square \phi
\end{aligned}
$$

By exploring the trace of the tensorial equation to eliminate the curvature scalar, one obtains a Klein-Gordon equation with the trace of the stress-energy tensor as a source:

$$
\square \phi=\frac{8 \pi}{3+2 \omega} T .
$$

It has been claimed that GR is recovered for the large $\omega$ limit [35]. The GR limit of the Brans-Dicke theory, however, is intricate, reducing to GR in the $\omega \rightarrow \infty$ limit only if the trace of the matter energy-momentum tensor does not vanish. Indeed, in that particular case (including the vacuum) the asymptotic behaviour in $\omega$ is different [36]. This has been explained in [37] in terms of the differences in the conformal invariance group modifying $\omega$ in the two cases. In light of this analysis it is not trivial to prove whether the same limit applies for vacuum, nevertheless it has been assumed by analysing the Cassini probe data [38], and stringent constraint $\omega>40000$ was set in order the Brans-Dicke theory to survive the Solar System tests $[11,39]$.

The Euler-Lagrange expressions for the Brans-Dicke Lagrangian in the Jordan frame are given by

$$
\begin{aligned}
16 \pi E_{\mu v} & =-\frac{\omega}{\phi} \nabla_{\mu} \phi \nabla_{\nu} \phi+\frac{\omega}{2 \phi}(\nabla \phi)^{2} g_{\mu v}+\phi G_{\mu v}-\nabla_{\mu} \nabla_{\nu} \phi+\square \phi g_{\mu v}, \\
16 \pi \phi E^{\phi} & =-\frac{\omega}{\phi} \nabla^{\mu} \phi \nabla_{\mu} \phi+\phi R+2 \omega \square \phi .
\end{aligned}
$$

Only the last three terms of $E_{\mu v}$ contain second derivatives, so only those will contribute singular terms. 
Due to smoothness in the domains $M^{ \pm}$, and continuity through $\Sigma$, the jump in the derivatives of $g_{\mu \nu}$ and $\phi$ are necessarily transversal [18]:

$$
\begin{aligned}
{\left[\partial_{\sigma} g_{\mu v}\right] } & =\eta n_{\sigma} c_{\mu v} \\
{\left[\partial_{\mu} \phi\right] } & =\eta n_{\mu} J .
\end{aligned}
$$

With these, the singular part of the Einstein-tensor can be expressed [18] as

$$
\mathscr{G}_{\mu \nu}=\frac{1}{2} \eta\left(n_{\mu} c_{\nu}+n_{\nu} c_{\mu}-n_{\mu} n_{\nu} c-g_{\mu \nu} c^{\dagger}-\epsilon\left(c_{\mu \nu}-c g_{\mu \nu}\right)\right),
$$

where we introduced the notations

$$
c_{\mu}=c_{\mu v} n^{v}, c=c^{\mu} \mu, c^{\dagger}=c_{\mu v} n^{\mu} n^{v} .
$$

One may easily check that $\mathscr{G}^{\mu v}$ is tangential $\left(\mathscr{G}^{\mu v} n_{v}=0\right)$; thus, it is possible to represent it as an intrinsic $\Sigma$-tensor as

$$
\mathscr{G}^{\mu \nu}=\mathscr{G}^{a b} e_{a}^{\mu} e_{b}^{v},
$$

this representation being invariant with respect to the choice of transversal vector $l^{\mu}$.

We find the singular part of the expression $\nabla_{\mu} \nabla_{\nu} \phi-\square \phi g_{\mu \nu}$ as

$$
J \eta\left(n_{\mu} n_{v}-\epsilon g_{\mu v}\right) \text {. }
$$

A contraction with $n^{v}$ reveals that this term is also tangential.

The tensorial junction Equation (11) then reads

$$
\begin{aligned}
8 \pi \mathscr{T}^{\mu v} & =\frac{1}{2} \phi \eta\left(n^{\mu} c^{v}+n^{v} c^{\mu}-n^{\mu} n^{v} c-g^{\mu v} c^{\dagger}-\epsilon\left(c^{\mu v}-c g^{\mu \nu}\right)\right) \\
& -J \eta\left(n^{\mu} n^{v}-\epsilon g^{\mu v}\right) .
\end{aligned}
$$

In what follows we will express this equation as an intrinsic $\Sigma$-tensor equation.

A convenient basis of the tangent spaces of $M$ along $\Sigma$ is $\left(l^{\mu}, e_{a}^{\mu}\right)$, with the dual frame $\left(\eta n_{\mu}, \theta_{\mu}^{a}\right)$, where $\theta^{a}{ }_{\mu}$ obeys the relations

$$
\theta_{\mu}^{a} e_{b}^{\mu}=\delta_{b}^{a}, \quad \theta_{\mu}^{a} l^{\mu}=0 .
$$

Unlike $e_{a}^{\mu}$, the covector fields $\theta_{\mu}^{a}$ depend on the choice of $l^{\mu}$. For any vector $X^{\mu}$ along $\Sigma$, the contraction $\theta_{\mu}^{a} X^{\mu}$ provides the tangential components of $X$. Further, when $X$ is purely tangential, this contraction simply provides its $l^{\mu}$-independent components in the coordinate frame adapted to $\Sigma$. This will be explored in identifying $\mathscr{E}^{a b}$ from $\mathscr{E}^{\mu v}$, when $\mathscr{E} a=0=\mathscr{E}$, and rewriting Equation (23) accordingly. In doing so we will explore the jump of the extrinsic curvature.

The extrinsic curvature of a space-like or time-like surface with normal $n^{\mu}$ is defined as $K_{a b}=\frac{1}{2} e_{a}^{\mu} e_{b}^{v} \mathscr{L}_{n} g_{\mu v}$. For null hypersurfaces this quantity does not carry transverse information (as $n^{\mu}$ becomes tangential), hence we replace it with the analogous transverse curvature [18]

$$
\mathscr{K}_{a b}=\frac{1}{2} e^{\mu} e^{\nu}{ }_{b}^{v} \mathscr{L}_{l} g_{\mu \nu} .
$$

The jump of the transverse curvature is

$$
\left[\mathscr{K}_{a b}\right]=\frac{1}{2} e_{a}^{\mu} e^{v}{ }_{b} c_{\mu v} \equiv \frac{1}{2} c_{a b} .
$$


To show how this relates to the full $c_{\mu v}$, we decompose the latter with respect to the dual frame

$$
c_{\mu v}=c^{n} n_{\mu} n_{v}+2 c_{a}^{n} n_{(\mu} \theta_{v)}^{a}+2\left[\mathscr{K}_{a b}\right] \theta^{a}{ }_{\mu} \theta_{v}^{b} .
$$

We also decompose the inverse metric with respect to the vector frame

$$
g^{\mu v}=\epsilon \eta^{2} l^{\mu} l^{v}+2 \eta n^{a} l^{(\mu} e_{a}^{v)}+h_{*}^{a b} e_{a}^{\mu} e_{b}^{v},
$$

where $n^{a}=n^{\mu} \theta_{\mu}^{a}$ is the "tangential" part of $n$ in the $n^{\mu}=n_{l} l^{\mu}+n^{a} e_{a}^{\mu}$ decomposition, and $h_{*}^{a b}$ is a pseudo-inverse metric on $\Sigma$, defined as $h_{*}^{a b}=g^{\mu v} \theta_{\mu}^{a} \theta_{v}^{b}$ (it becomes the inverse for non-null hypersurfaces if the transverse is chosen as the normal).

Because Equation (23) is tangential (as can be seen by contracting with $n_{\mu}$ ), contracting with $\theta_{\mu}^{a} \theta_{v}^{b}$ gives the intrinsic components of the tensorial junction condition

$$
\begin{aligned}
\mathscr{T}^{a b} & =\frac{\phi \eta}{8 \pi}\left[\mathscr{K}_{c d}\right]\left(h_{*}^{a c} n^{b} n^{d}+h_{*}^{b c} n^{a} n^{d}-h_{*}^{c d} n^{a} n^{b}-h_{*}^{a b} n^{c} n^{d}\right. \\
& \left.-\epsilon\left(h_{*}^{a c} h_{*}^{b d}-h_{*}^{a b} h_{*}^{c d}\right)\right)-\frac{J \eta}{8 \pi}\left(n^{a} n^{b}-\epsilon h_{*}^{a b}\right)
\end{aligned}
$$

This is the analogue of the Lanczos equation of GR and it is valid for arbitrary junction surface $\Sigma$.

The non-null GR limit is readily obtained with $\phi=G^{-1}$ and $J=0$, and further simplified by the choice $l^{\mu}=n^{\mu}$. This gives $n^{a}=0, \eta=\epsilon, \mathscr{K}_{a b}=K_{a b}$ and $h_{*}^{a b}=h^{a b}$. Inserting these into Equation (29) gives

$$
\mathscr{T}^{a b}=-\frac{1}{8 \pi G}\left(\left[K^{a b}\right]-[K] h^{a b}\right),
$$

the familiar Lanczos equation of GR.

Next we consider the scalar junction condition (11). In the scalar equation of motion (15) only the terms $\phi R+2 \omega \square \phi$ contain second derivatives, only they contribute the singular parts

$$
\mathscr{E}_{\phi}=\frac{1}{16 \pi}\left(\phi \eta\left(c^{\dagger}-\epsilon c\right)+2 \omega \eta \epsilon J\right)
$$

As a scalar equation, this is already intrinsic to $\Sigma$. Proceeding as in the case of the tensorial equation, we explore Equation (27) to express $c^{\dagger}$ and $c$ in terms of $c^{n}, c_{a}^{n}$ and $c_{a b}=2\left[\mathscr{K}_{a b}\right]$. After simplification and inserting into $\mathscr{E}_{\phi}=0$ we get

$$
0=\phi \eta\left[\mathscr{K}_{a b}\right]\left(n^{a} n^{b}-\epsilon h_{*}^{a b}\right)+2 \omega \eta \epsilon J
$$

This, together with Equation (29) constitute the junction equations in Brans-Dicke theory.

The non-null limit (with the choice $l^{\mu}=n^{\mu}$ and consequences as described earlier) of these junction conditions arises as

$$
\begin{aligned}
\mathscr{T}^{a b} & =-\frac{\phi}{8 \pi}\left(\left[K^{a b}\right]-[K] h^{a b}-J h^{a b}\right), \\
0 & =-\frac{1}{8 \pi}(\phi[K]-2 \omega J) .
\end{aligned}
$$

These junction conditions derived in the Jordan frame correspond to the one scalar case of those obtained in Einstein frame in the context of multi-scalar tensor theories of gravity in [14].

\section{The Null Case}

The junction equations derived so far are applicable to any hypersurface, irrespective of its causal character. In what follows, we assume $\Sigma$ a null hypersurface, so the normal vector becomes also tangential. Hence the choice $l^{\mu}=n^{\mu}$ leads to a degenerate situation, and another simplifying 
assumption for the transverse vector should be made. This is covered by our forthcoming analysis, which in turn closely follows the one presented for GR by Poisson [40]. We will chose an autoparallel normal vector, denoted $N^{\mu}$ (satisfying $N^{\nu} \nabla_{v} N^{\mu}=\kappa N^{\mu}$ ) and we will use a coordinate system adapted to $N^{\mu}$. The parameter along the integral curves of $N^{\mu}$ is $\lambda$, one of the coordinates on $\Sigma$. We denote the two additional coordinates $\left\{\sigma^{A}\right\}$ (capital latin indices taking the values 2,3), labelling the geodesic integral curves of $N^{\mu}$. The transverse vector, denoted $L^{\mu}$ is chosen $[40,41]$ as

$$
L_{\mu} L^{\mu}=0, L^{\mu} N_{\mu}=1, L_{\mu} e_{A}^{\mu}=0 .
$$

Here $e_{A}^{\mu}=\partial x^{\mu} / \partial \sigma^{A}$ are the coordinate basis fields associated to $\sigma^{A}$. If $e_{A}^{\mu}$ also obey orthonormality relations, the basis (35) is pseudoorthonormal. The induced metric in this basis is manifestly two-dimensional:

$$
h_{11}=g_{\mu v} N^{\mu} N^{v}=0, h_{1 A}=g_{\mu v} N^{\mu} e_{A}^{v}=0, q_{A B} \equiv h_{A B}=g_{\mu v} e_{A}^{\mu} e_{B}^{v} .
$$

It is easy to check that $q_{A B}$ (as opposed to $h_{a b}$ ) is non-degenerate, and a unique inverse $q^{A B}$ exists. If we define the dual frame $e_{\mu}^{A}=g_{\mu \nu} q^{A B} e_{B}^{v}$, it obeys

$$
e_{\mu}^{A} e_{B}^{\mu}=q_{B C} q^{A C}=\delta_{B}^{A} .
$$

The dual frame of $\left(L^{\mu}, N^{\mu}, e_{A}^{\mu}\right)$ is $\left(N_{\mu}, L_{\mu}, e_{\mu}^{A}\right)$.

With it the components of the normal (defined as $n^{a}=n^{\mu} \theta_{\mu}^{a}$ ) become $N^{\lambda}=N^{\mu} L_{\mu}=1$ and $N^{A}=N^{\mu} e_{\mu}^{A}=0$, hence $n^{a}=\delta_{\lambda}^{a}$. The normal $n^{a} \equiv e_{\lambda}^{a}=\delta_{\lambda}^{a}$ is therefore a first basis vector on the tangent space of $\Sigma$, the other two being $e^{a}{ }_{A}=\delta^{a}{ }_{A}$. The pseudo-inverse metric has the components

$$
h_{*}^{\lambda \lambda}=g^{\mu v} L_{\mu} L_{v}=0, h_{*}^{\lambda A}=g^{\mu v} L_{\mu} e_{v}^{A}=0, h_{*}^{A B}=g^{\mu v} e_{\mu}^{A} e_{v}^{B}=q^{A B} .
$$

By inserting these into Equation (29) and substituting $\eta=1$ and $\epsilon=0$, we obtain the tensorial junction condition

$$
\mathscr{T}^{a b}=\rho e_{\lambda}^{a} e_{\lambda}^{b}+j^{A}\left(e^{a}{ }_{A} e^{b}{ }_{\lambda}+e^{a}{ }_{\lambda} e^{b}{ }_{A}\right)+p q^{A B} e^{a}{ }_{A} e_{B}^{b},
$$

where

$$
\rho=-\frac{\phi}{8 \pi}\left[\mathscr{K}_{A B}\right] q^{A B}-\frac{J}{8 \pi}
$$

is the surface density of the layer,

$$
j^{A}=\frac{\phi}{8 \pi}\left[\mathscr{K}_{B \lambda}\right] q^{A B}
$$

the surface current of the layer, and

$$
p=-\frac{\phi}{8 \pi}\left[\mathscr{K}_{\lambda \lambda}\right]
$$

the isotropic pressure of the layer. With $\phi=G^{-1}$ and $J=0$ the corresponding result derived in [40] is reobtained.

Finally the scalar junction Equation (32) simplifies to $0=\phi\left[\mathscr{K}_{\lambda \lambda}\right]$, implying that

$$
p=0,
$$

thus, the presence of a continuous scalar field contributing to the expressions of the surface density, current and pressure does not allow for an isotropic surface pressure on the null layer. This result is consistent with the pressurelessness condition derived in [14] for a multiscalar generalization of Brans-Dicke theory in the Einstein frame. 


\section{Conclusions}

The junction of space-time regions with different geometrical characteristics is an important task in all geometric theories of gravity. It is of particular interest when the separating hypersurface is singular, carrying distributional energy-momentum tensor. The junction formalism is complicated if the hypersurface is null. We have derived the generic junction conditions for Brans-Dicke theory in the Jordan frame, as this is the frame in which the matter energy-momentum conservation holds (the physical frame). We explored a formalism based on a transverse vector, rather than normal, which can be applied to any type of hypersurface. Then we considered the particular cases of (i) non-null hypersurfaces, obtaining the generalisations of the Lanczos equation, in which the jump of the extrinsic curvature is sourced by both the distributional energy-momentum tensor and by the jump in the transverse derivative of the scalar, and ii) null hypersurfaces, which represent shock-waves propagating with the speed of light. In the latter case the distributional source is decomposed into surface density, current and pressure. The latter, however, ought to vanish by virtue of the scalar junction condition. A similar result derived as a traceless requirement for the distributional energy-momentum source in the Einstein frame is a remarkable example of the frame independence of a physical result.

Confronting these results with previous ones derived for non-null hypersurfaces in the Einstein frame, as well as their generalisation for the so-called Generalised Brans-Dicke theory (given by the Lagrangian $L_{G B D}=\frac{1}{2} F(\phi) R+B(\phi) X-2 G(\phi) \square \phi X$, where $F, B, G$ are arbitrary smooth functions of $\phi$ and $X$ are in progress and will be presented elsewhere.

Acknowledgments: L.Á.G. thanks Shinji Mukohyama for inspiring discussions on the topic. This work was supported by the Hungarian National Research Development and Innovation Office (NKFI) in the form of the grant 123996. The authors thank the organisers of the Bolyai-Gauss-Lobachevsky Conference for partial support of their participation.

Author Contributions: All authors contributed equally to this work.

Conflicts of Interest: The authors declare no conflict of interest.

\section{References}

1. LIGO Scientific Collaboration; Virgo Collaboration. Observation of Gravitational Waves from a Binary Black Hole Merger. Phys. Rev. Lett. 2016, 116, 061102.

2. LIGO Scientific Collaboration; Virgo Collaboration. GW151226: Observation of Gravitational Waves from a 22-Solar-Mass Binary Black Hole Coalescence. Phys. Rev. Lett. 2016, 116, 241103.

3. LIGO Scientific Collaboration; Virgo Collaboration. GW170104: Observation of a 50-Solar-Mass Binary Black Hole Coalescence at Redshift 0.2. Phys. Rev. Lett. 2017, 118, 221101.

4. LIGO Scientific Collaboration; Virgo Collaboration. GW170608: Observation of a 19-Solar-Mass Binary Black Hole Coalescence. Astrophys. J. Lett. 2017, 851, L35.

5. LIGO Scientific Collaboration; Virgo Collaboration. GW170814: A Three-Detector Observation of Gravitational Waves from a Binary Black Hole Coalescence. Phys. Rev. Lett. 2017, 119, 141101.

6. LIGO Scientific Collaboration; Virgo Collaboration. GW170817: Observation of Gravitational Waves from a Binary Neutron Star Inspiral. Phys. Rev. Lett. 2017, 119, 161101.

7. LIGO Scientific Collaboration; Virgo Collaboration. Tests of General Relativity with GW150914. Phys. Rev. Lett. 2016, 116, 221101.

8. LIGO Scientific Collaboration; Virgo Collaboration. Fermi Gamma-ray Burst Monitor, and INTEGRAL, Gravitational Waves and Gamma-Rays from a Binary Neutron Star Merger: GW170817 and GRB170817A. Astrophys. J. Lett. 2017, 848, L13.

9. Horndeski, G.W. Second-order scalar-tensor field equations in a four-dimensional space. Int. J. Theor. Phys. 1974, 10, 363-384.

10. Deffayet, C.; Gao, X.; Steer, D.A.; Zahariade, G. From k-essence to generalized Galileons. Phys. Rev. D 2011, 84,064039 . 
11. Capozziello, S.; Faraoni, V. The variational principle and the field equations of Brans-Dicke gravity. In Beyond Einstein Gravity: A Survey of Gravitational Theories for Cosmology and Astrophysics; Springer: Berlin, Germany, 2011; pp. 59-61.

12. Dicke, R.H. Mach's principle and invariance under transformation of units. Phys. Rev. 1962, 125, $2163-2167$.

13. Faraoni, V.; Nadeau, S. The (pseudo)issue of the conformal frame revisited. Phys. Rev. D. 2007, 75, 023501.

14. Barrabès, C.; Bressange, G.F. Singular hypersurfaces in scalar-tensor theories of gravity. Class. Quantum Gravity 1997, 14, 805-824.

15. Faraoni, V., Gunzig, E. Einstein frame or Jordan frame? Int. J. Theor. Phys. 1999, 38, 217-225.

16. Magnano, G.; Sokolowski, L.M. On Physical equivalence between nonlinear gravity theories and a general-relativistic self-gravitating scalar field. Phys. Rev. D. 1994, 50, 5039-5059.

17. Israel, W. Singular hypersurfaces and thin shells in general relativity. Nuovo Cim. B 1966, 44, 1-14.

18. Barrabès, C.; Israel, W. Thin shells in general relativity and cosmology: The lightlike limit. Phys. Rev. D 1991, 43, 1129-1142.

19. Mukohyama, S. Doubly covariant action principle of singular hypersurfaces in general relativity and scalar-tensor theories. Phys. Rev. D 2001, 65, 024028.

20. Schmidt, H.; Wang, A. Plane domain walls when coupled with the Brans-Dicke scalar field. Phys. Rev. D 1993, 47, 4425-4432.

21. Letelier, P.S.; Wang, A. Spherically symmetric thin shells in Brans-Dicke theory of gravity. Phys. Rev. D 1993, $48,631-646$.

22. Sakai, N.; Maeda, K. Bubble dynamics and space-time structure in extended inflation. Phys. Rev. D 1993, 48, 5570-5575.

23. Sakai, N.; Maeda, K. Bubble dynamics in generalized Einstein theories. Prog. Theor. Phys. 1993, 90, 1001-1018.

24. Padilla, A.; Sivanesan, V. Boundary terms and junction conditions for generalized scalar-tensor theories. J. High Energy Phys. 2012, 2012, 122.

25. Nishi, S.; Kobayashi, T.; Tanahashi, N.; Yamaguchi, M. Cosmological matching conditions and galilean genesis in Horndeski's theory. J. Cosmol. Astropartic. Phys. 2014, 2014, 008.

26. Geroch, R.; Traschen, J.H. Strings and Other Distributional Sources in General Relativity. Phys. Rev. D 1987, $36,1017-1031$.

27. Clarke, C.J.S.; Dray, T. Junction conditions for null hypersurfaces. Class. Quantum Gravity 1987, 4, $265-275$.

28. Ostrogradsky, M. Mémoires sur les équations différentielles, relatives au problème des isopérimètres. Mem. Acad. St. Petersbourg 1850, 6, 385-517.

29. Woodard, R.P. The Theorem of Ostrogradsky. arXiv 2015, arXiv:1506.02210.

30. Kobayashi, T.; Yamaguchi, M.; Yokoyama, J.; Generalized G-inflation: Inflation with the most general second-order field equations. Prog. Theor. Phys. 2011, 126, 511-529.

31. De Felice, A.; Tsujikawa, S. Conditions for the cosmological viability of the most general scalar-tensor theories and their applications to extended Galileon dark energy models. J. Cosmol. Astrophys. Phys. 2012, 2012, 007.

32. Baker, T.; Bellini, E.; Ferreira, P.G.; Lagos, M.; Noller, J.; Sawicki, I. Strong constraints on cosmological gravity from GW170817 and GRB 170817A. Phys. Rev. Lett. 2017, 119, 251301.

33. Ezquiaga, J.M.; Zumalacarregu, M. Dark Energy after GW170817: Dead ends and the road ahead. Phys. Rev. Lett. 2017, 119, 251304.

34. Creminelli, P.; Vernizzi, F. Dark Energy after GW170817 and GRB170817A. Phys. Rev. Lett. 2017, 119, 251302.

35. Weinberg, S. The Brans-Dicke theory. In Gravitation and Cosmology; John Wiley \& Sons Inc.: Hoboken, NJ, USA, 1972; pp. 157-160.

36. Banerjee N.; Sen, S. Does Brans-Dicke theory always yield general relativity in the infinite $\omega$ limit? Phys. Rev. D 1997, 56, 1334-1337.

37. Faraoni, V. The $\omega \rightarrow \infty$ limit of Brans-Dicke theory. Phys. Lett. A 1998, 245, 26-30.

38. Bertotti, B.; Iess, L.; Tortora, P. A test of general relativity using radio links with the Cassini spacecraft. Nature 2003, 425, 374-376.

39. Freire, P.C.C.; Wex, N.; Esposito-Farése, G.; Verbiest, J.P.W.; Bailes, M.; Jacoby, B.A.; Kramer, M.; Stairs, I.H.; Antoniadis, J.; Janssen, G.H. The relativistic pulsar-white dwarf binary PSR J1738+0333-II. The most stringent test of scalar-tensor gravity. Mon. Not. R. Astron. Soc. 2012, 423, 3328-3343. 
40. Possion, E. Null Shells. In A Relativist's Toolkit: The Mathematics of Black-Hole Mechanics; Cambridge University Press: Cambridge, UK, 2004; pp. 98-104.

41. Hawking, S.W.; Ellis, G.F.R. Null curves. In The Large-Scale Structure of Spacetime; Cambridge University Press: Cambridge, UK, 1973; pp. 86-87.

(C) 2018 by the authors. Licensee MDPI, Basel, Switzerland. This article is an open access article distributed under the terms and conditions of the Creative Commons Attribution (CC BY) license (http:/ / creativecommons.org/licenses/by/4.0/). 